\title{
Huxley's Model of Muscle Contraction with Compliance
}

\author{
W. O. Williams
}

January 3, 2011

\begin{abstract}
Huxley's cross-bridge dynamics of muscle contraction is widely used in understanding, in particular, laboratory experiments on muscles and subunits of muscle. The hard-connection version of the model has several defects. In this paper I present a detailed and precise method of solution of the problem with a compliant element in series with the muscle.
\end{abstract}

\section{Introduction}

The Huxley [4] model of muscle contraction, formulated more than fifty years ago, still is the model most used by experimenters. This fact reflects both the simplicity of the model and the inability of more elaborate models simultaneously to replicate its precision on the basic experimental tests and to apply successfully to more than a single extended test.

The formulation is a simple population-dynamics model, with elementary affine birth- and death-rate rules and a linear elastic mechanics for force generation. Various proposals to "tweak" the system by altering these rules have not greatly improved its predictive ability, in particular not repairing defects in its predictions (including those noted by Huxley himself).

One aspect of the Huxley model which hampers its performance is that it is a "hard" system, with rigid attachment of the force-generating element to attachment points. Of course all physiologists recognize the elasticity of tendon and the microscopic fibers within the muscle itself, and a few adjusted models have appeared in the literature. In this paper, I derive the equations which describe the system with an added serial elastic element and give a formal integration scheme for them. I present computations to verify that the equations can predict two classical experimental results not within the scope of the original forms. 


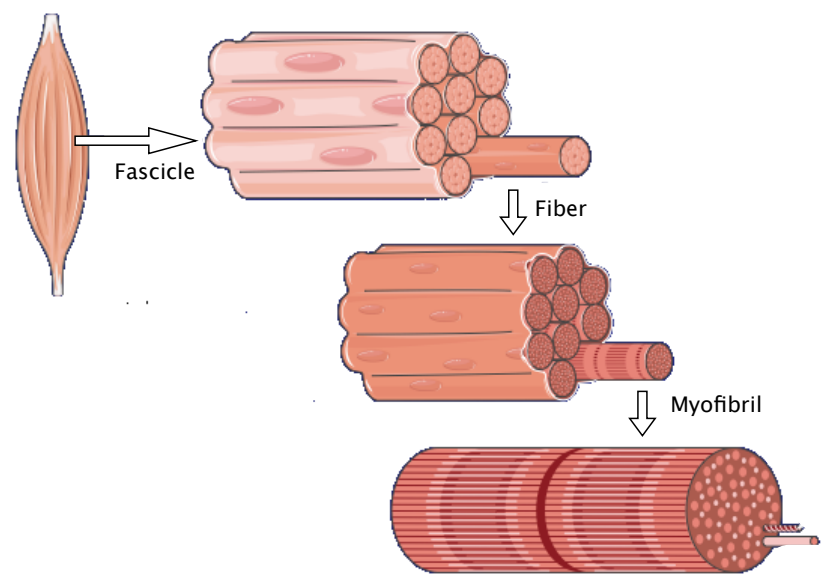

Figure 1: An illustration of the structure of a skeletal muscle

\section{Physiology and Experiments}

Figure 1 illustrates the base structure of skeletal (striated) muscle, as observed since the invention of the light microscope. The remarkable fact which enables extension of calculations from molecular level to (nearly) whole muscle is the regularity of the structure. Each muscle is comprised of a collection of fascicles, usually laid in a parallel arrangement, and each of these fascicles is a bundle of muscle fibres likewise laid in a parallel arrangement. To have a sense of scale: the fibres, each of which is a single cell, may be 10-100 $\mu$ in diameter, while being as much as $30 \mathrm{~cm}$ long. There may be 100,000 fibres in a muscle. The regularity of structure extends to the next level as well. As sketched, each fibre includes a bundle of a hundred or more myofibrils, approximately 1-3 $\mu$ in diameter. The myofibrils, as illustrated, have a banded appearance, leading to the description of skeletal muscle as striated muscle. This regular striation consists in periodic structures called sarcomeres (Figure 2).

Each sarcomere is about $2.5 \mu$ long and is symmetric. Within the sarcomere there are, as indicated, filaments, which are coiled collections of polymeric chains of molecules. The thicker ones are myosin; the regular array of thick filaments is interleaved in a regular geometric pattern with thin filaments of actin molecules, each thick filament surrounded by six thin ones. The myosin filaments are anchored at each end of the sarcomere; the actin filaments are attached together by a membrane in the center. The thick filaments are approxi- 


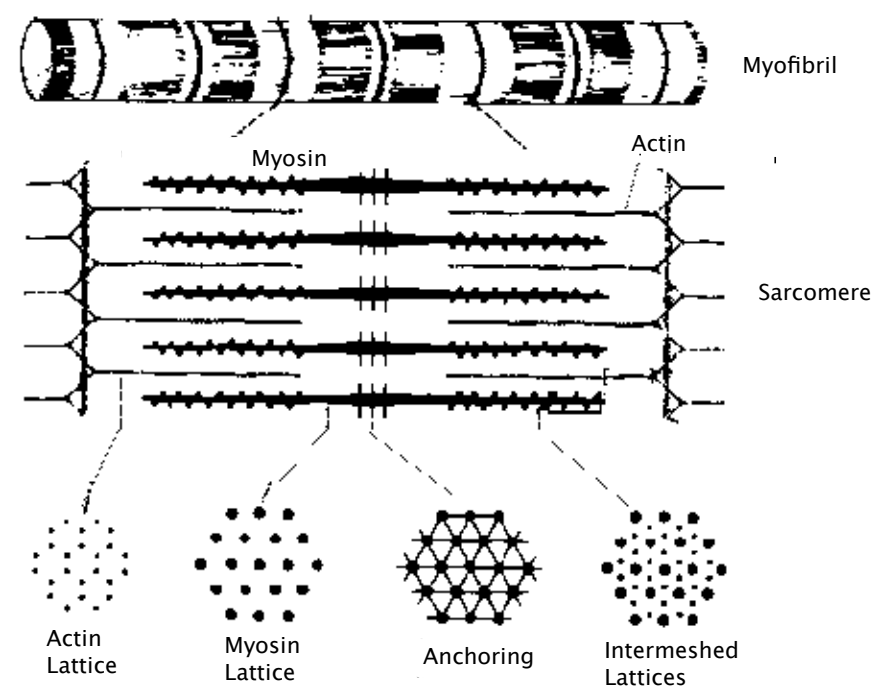

Figure 2: The sarcomere.

mately $15 \mathrm{~nm}$ in diameter, the thin ones about $6 \mathrm{~nm}$. Because of the symmetry of the sarcomere, the computational unit which we consider is the half-sarcomere.

Contraction of the muscle is effected by sliding of the thin and the thick filaments past one another. The mechanism that engenders this sliding action is now agreed to be the action of the myosin cross-bridges. Each myosin molecule consists of a long thin tail with a pair of globular heads. About 180 of these molecules are coiled together to form a thick filament with the heads and the leading part of the tail projecting from the coil to form a cross-bridge. The heads include a binding site for actin, and a site which accepts adenosine triphosphate, or ATP, molecules. The thin filament, in turn, consists of two polymeric chains of actin in a helical coil which presents spaced binding sites for the myosin.

When the muscle is stimulated, in vivo through the presence of $\mathrm{Ca}^{2+}$ ions perfusing the sarcomere and unblocking binding sites on the actin filament, the cross-bridge head attaches to the adjacent thin filament. At this point hydrolization of an attached ATP molecule (which loses a phosphate and becomes adenesine diphosphate or ADP) creates a change in angle of the myosin head relative its tail, and the result is a shift of the cross-bridge head's pivot point relative the thin filament. At the end of the process the myosin head detaches (with another ATP-ADP reaction).

The angular shift can have two results (or a combination of the two) in that 
the thin filaments may slide past the thick ones, or, if constrained from so doing, the shift stretches the elastic cross-bridge tail (and the compliant actin and myosin filaments). The stretching generates the force which the muscle exerts, while the sliding generates the contraction of the muscle.

Modeling of the behavior of larger units, filament, fascicle or whole muscle is deduced from the behavior of sarcomeres, due to the regular arrangement of the elements, since it is usually held that the stimulation is essentially simultaneous throughout the muscle. The parallel arrangement of fibrils leads to additivity of forces across the cross-section, extending to the entire muscle. Likewise, the concatenation of sarcomeres engenders additivity of length, and hence additivity of velocity along the length of a fibre. That neither additivity is absolutely true in detail has been observed ${ }^{1}$ but in any case additivity seems a reasonable assumption.

There are several classical experiments which serve as benchmarks for any proposed model. We look at the ones which are most relevant to our purposes here.

A twitch is the result of a single pulse of stimulation applied to a muscle. Normally the experiment is done with the muscle held at fixed length and the resulting force measured. The force ascends to a peak value and then decays to zero.

A tetanus is the result of a sustained stimulation. Again the standard experiment has the muscle held at fixed length, with force measured. A typical trace, with a twitch of the same muscle, is shown in Figure 3

A later result is important to us: experiments show that in a tetanus, the force evolution toward its maximum lags behind the evolution of the number of attached cross-bridges. (The latter is deduced from measurements of the evolution of the stiffness of the muscle.) This lagging of force is contrary to the predictions of the simplest version of the Huxley model.

\section{Basics of Huxley's Model.}

In 1957 A. E. Huxley [4] developed the base model which has proved the foundation for all subsequent work, including elaborations which he shared in creating. Here we examine the ideas basic to this model. It is important to note that this model pre-dates the notion of rotation of the myosin heads; in fact, at the time it was considered debatable even that the cross-bridges were the actors in gener-

\footnotetext{
${ }^{1}$ Perreqault et al [32] find non-additivity of force. It has long been suggested that length changes may not be uniform along the muscle; see, e.g., Sugi and Kobayashi [10], See also the discussion by Zajac [15].
} 


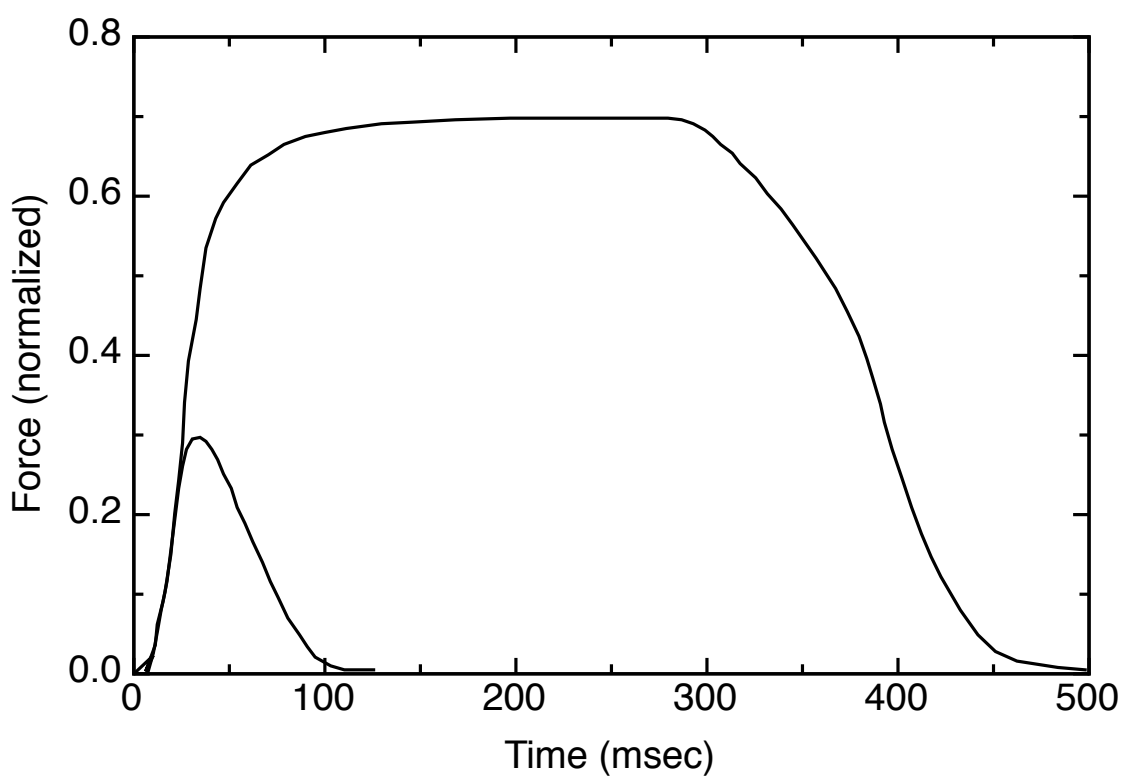

Figure 3: Tetanus and twitch traces superposed, using data from [30] .

ating the relative sliding of the two classes of fibers; Huxley proposed the model to aid in validating this concept ${ }^{2}$

Consider a half-sarcomere. Huxley pictured the myosin heads, attached to the parent myosin filament by elastic tails, by utilizing a cartoon replicated in Figure 4. When the muscle is stimulated, those heads which are in the vicinity of an attachment site on the actin filament can be expected to attach to that site. Force then would be applied to the actin filament by the stretched elastic tail of the cross-bridge; a contractile force being created if the elastic tail is in a state of extension. Since a contraction velocity would tend to shorten the elastic tail, in order that force be created one must suppose that the cross-bridge tail already is extended when the cross-bridge attaches. Huxley proposed that this extension could be provided by thermal agitation of the cross-bridge head. Since this agitation should be as likely to contract as to extend the tails of individual cross-bridges, Huxley further suggested that attachment would, by an unspecified chemo-mechanical mechanism, be facilitated for the cross-bridges which are displaced positively and made difficult for those whose tails are in a

\footnotetext{
${ }^{2}$ The cross-bridge-generated sliding-filament model was not universally accepted until the invention of the electron microscope enabled direct observation.
} 


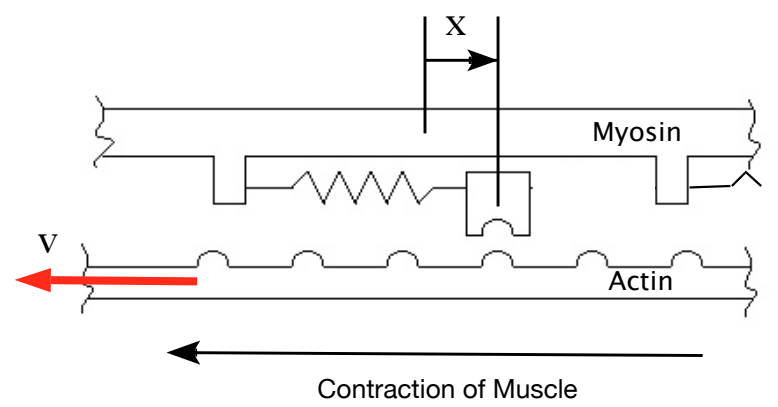

Figure 4: Huxley's basic cartoon

rest position or contracted $\mathrm{H}^{3}$

The variable of significance for the problem is the displacement $x$. Based then on the enormous numbers of cross bridges involved, it is reasonable to model the number of cross-bridges as a distribution in $x$. The number of crossbridges which are attached to the actin filament at time $t$ and which have displacement between $a$ and $b$ is

$$
N((a, b), t)=\int_{a}^{b} n(x, t) \mathrm{d} x
$$

and the total number attached is

$$
N(t)=\int_{-X}^{X} n(x, t) \mathrm{d} x
$$

where $X$ is the maximum possible extension of the cross-bridge tail.

The balance relation for numbers of cross-bridges in the interval $(a, b)$ will involve an attachment rate $F(x, t)$ and a detachment rate $G(x, t)$ and also transport effects, as the velocity of contraction, in drawing the actin filament past the myosin filament, will carry cross-bridges into and out of this range of extension. We suppose that both $F$ and $G$ are piecewise continuous and of compact support. Then we have

$$
\begin{aligned}
\frac{d}{d t} N((a, b), t) & =\int_{a}^{b} \frac{\partial n}{\partial t}(x, t) \mathrm{d} x \\
& =\int_{a}^{b}(F(x, t)-G(x, t)) \mathrm{d} x+v(t)[n(b, t)-n(a, t)] .
\end{aligned}
$$

\footnotetext{
${ }^{3}$ Huxley recognized the ad hoc nature of these suppositions, and ultimately revised the model.
} 
The signs on the latter terms reflect the fact that the velocity of contraction $v(t)$ carries cross-bridges with a certain displacement into smaller values of displacement. If we divide this equation by $b-a$ and take the limit as $b$ approaches $a$, we obtain the equation of balance of cross-bridges:

$$
\frac{\partial n(x, t)}{\partial t}=F(x, t)-G(x, t)+v(t)\left(\frac{\partial n(x, t)}{\partial x}\right)
$$

or

$$
\frac{\partial n(x, t)}{\partial t}-v(t)\left(\frac{\partial n(x, t)}{\partial x}\right)=F(x, t)-G(x, t)
$$

The natural associated initial condition would be

$$
n(x, 0)=n_{o}(x)
$$

Following the Huxley cartoon, each attached cross-bridge provides a force according to the extension imposed upon the cross-bridge's tail section. Supposing that the response is elastic ${ }^{4}$, we arrive at the form

$$
P(t)=\int_{-X}^{X} \mathscr{E}(x) n(x, t) \mathrm{d} x .
$$

Here $P(t)$ is the tension of the muscle, and we suppose $\mathscr{E}$ to be an non-decreasing function, zero at $x=0$. It seems to be most consistent with the physical model to suppose that negative values of $x$ should produce no compressive force, although compressive forces are allowed in some elaborations of the model.

Although it is generally recognized that biological materials are generically non-linear in response, Huxley, and most of the developers of his model up to the current time, assume the elasticity in the myosin tails to be linear. In part, this is justified by convenience and in part by recognition that in light of the large numbers involved an averaged elasticity may be appropriate.

Two steps remain before we introduce the particular constitutive equations of the Huxley 1957 model.

First, it is natural to simplify the form of the computations by normalizing the distribution functions. In light of the parallel nature of the structures, scaling upwards by the number of cross-bridges in an assembly then is quite simple. While it seems natural to normalize by dividing the density $n(x, t)$ by the total

\footnotetext{
${ }^{4}$ It has long been recognized that biological materials, and in particular, polymers like the actin filaments, display more elaborate behaviors (see, e.g., [37), in particular, both rate-dependence and some memory effects.
} 
number of cross-bridges available, in order to justify the linearization in the uptake and loss functions which is assumed by Huxley and all elaborators of his model, one should divide $n(x, t)$ by the total number of cross-bridges available at the extension $x$. Implicitly, Huxley and the others assume that the total population of cross-bridges at extension $x, U(x, t)$, is a constant, independent of both $x$ and $t t^{5}$. Let this constant be $U$; we define a new density

$$
w(x, t)=\frac{n(x, t)}{U} .
$$

and rewrite the balance equation:

$$
\frac{\partial w(x, t)}{\partial t}-v(t)\left(\frac{\partial w(x, t)}{\partial x}\right)=\frac{F(x, t)}{U}-\frac{G(x, t)}{U}
$$

Second, we note that it is convenient for analysis and computation to use a restatement of the problem by using characteristic coordinates. We define

$$
\chi(r, t):=r-\int_{0}^{t} \nu(s) \mathrm{d} s=: r-R(t)
$$

so that $r$ is a virtual reference displacement: one pictures $r=$ constant as identifying a cross-bridge attached and of length $r$ at time 0 so that $\chi$ tracks its history as time goes on. We use (9) to change coordinates via

$$
\sigma(r, t):=w(\chi(r, t), t),
$$

so that, for example, the total number of attached cross-bridges can be expressed as

$$
N(t)=U \int_{-\infty}^{\infty} \sigma(r, t) \mathrm{d} r
$$

of course the support of $\sigma(\cdot, t)$ is finite. This enables us to rewrite (4) as

$$
\frac{\partial \sigma}{\partial t}(r, t)=\frac{F(\chi(r, t), t)}{U}-\frac{G(\chi(r, t), t)}{U} .
$$

In his original article, Huxley chose to model the attachment and detachment rates for cross-bridges as

$$
\frac{F(x, t)}{U}=(1-w(x, t)) f(x)
$$

and

$$
\frac{G(x, t)}{U}=g(x) w(x, t)
$$




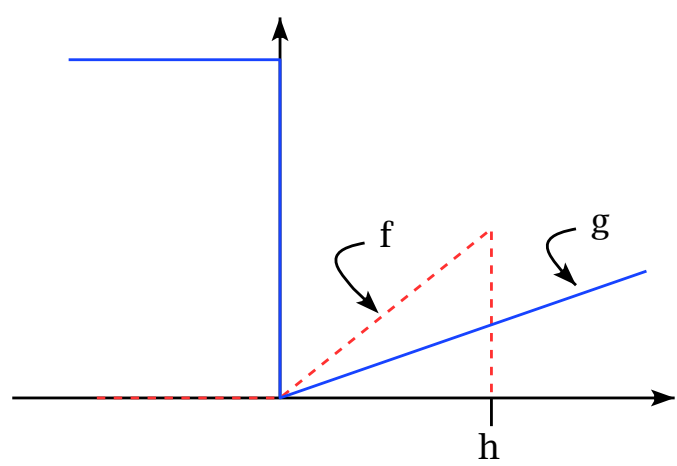

Figure 5: Huxley's attachment and detachment functions

He took $f$ and $g$ to be of the forms shown in Figure 5 note that $f$ is zero outside the interval $[0, h]$ while g continues indefinitely.

Huxley said that he assumed $f(x)$ and $g$ to be linear in positive displacement $x$ merely to simplify the analysis. The presence of the sharp limits on attachment rate, 0 and $h$, represent the idea that attachments which would work against the acceleration of the motion are blocked, and that the extension of the crossbridge tail is limited while the cross-bridge is unattached $\sqrt{6}$. We presume that $h \leq X$. Setting the detachment rate to be very high when the cross-bridge tails are forced into a negative extension is a soft version of a stripping rule. Growth of $g$ with positive extension reflects a susceptibility of cross-bridges to detachment when they are over-extended

Huxley's 1957 version of the balance equation 8 , then is

$$
\left.\begin{array}{rl}
\frac{\partial \sigma}{\partial t}(r, t) & =f(\chi(r, t))-[f(\chi(r, t))+g(\chi(r, t))] \sigma(r, t), \\
\sigma(r, 0) & =w_{0}(r) .
\end{array}\right\}
$$

Finally, to replicate Huxley's calculations, we assume that the cross-bridge tails are linearly elastic, so that the form of the force function now is

$$
P(t)=U E_{m} \int_{-X}^{X} x w(x, t) \mathrm{d} x=U E_{m} \int_{-\infty}^{\infty} \chi(r, t) \sigma(r, t) \mathrm{d} r .
$$

\footnotetext{
${ }^{5}$ That this is necessary to ensure consistency apparently first was noted by Keener and Sneyd 38, $c f .34$.

${ }^{6}$ While $h$ is thus proposed as the the maximal "natual" extension of a cross-bridge, it is not a maximum for extension, since a cross-bridge may be displaced further through a forced extension.
} 


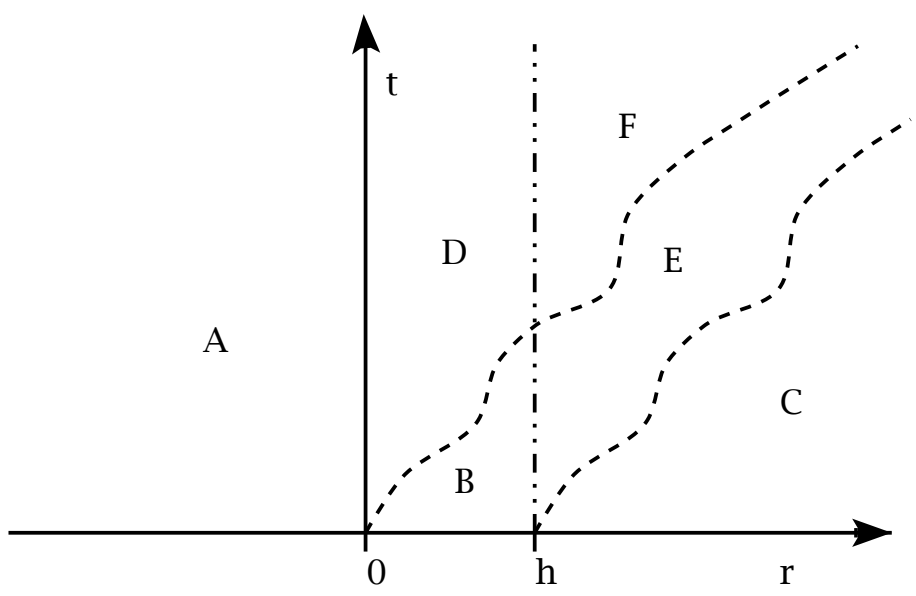

Figure 6: Characteristics (dashed lines) in the $r-t$ plane.

Note that a uniform delta-function increment in displacement would produce a delta-function increment in tension of

$$
U E_{m} \int_{-X}^{X} w(x, t) \mathrm{d} x=N(t) E_{m},
$$

yielding an instantaneous modulus of elasticity of the contractile unit.

To obtain explicit or easily computable solutions, we formally integrate the balance equation (15). Consider the $r-t$ plane as shown in Fig6. If we suppose that $v$ always is positive, then $R$ as defined in 9 is invertible and the graphs of $r=R(t)$ and $r=h+R(t)$ might look like those plotted as dashed lines. The vertical bars and these section the plane into the domains $\mathrm{A}, \mathrm{B}, \mathrm{C}, \mathrm{D}, \mathrm{E}$ and F, with slightly different formulae applying in each.

In $\mathrm{A}, \mathrm{D}$ and $\mathrm{F}$ we have $x \leq 0$ and thus solve

$$
\frac{\partial \sigma(r, t)}{\partial t}=-g_{2} \sigma(r, t)
$$

yielding

$$
\sigma(r, t)=K(r) \exp \left\{-g_{2} t\right\} .
$$

The initial condition, and hence $K(r)$, differs from region to region.

In $\mathrm{B}$ and $\mathrm{E}$ we have $0 \leq x \leq h$, so that we solve

$$
\frac{\partial \sigma(r, t)}{\partial t}=f_{1} \chi(r, t)-\left[f_{1}+g_{1}\right] \chi(r, t) \sigma(r, t)
$$


yielding

$$
\begin{aligned}
\sigma(r, t) & =f_{1} \int_{0}^{t}\left[\chi(r, s) \exp \left\{-\left(f_{1}+g_{1}\right) \int_{s}^{t} \chi(r, \tau) \mathrm{d} \tau\right\}\right] \mathrm{d} s \\
& +K(r) \exp \left\{-\left(f_{1}+g_{1}\right) \int_{0}^{t} \chi(r, s) \mathrm{d} s\right\} .
\end{aligned}
$$

Again, the initial condition differs between the two regions.

In $\mathrm{C}$ we solve

$$
\frac{\partial \sigma(r, t)}{\partial t}=-g_{1} \chi(r, t) \sigma(r, t)
$$

yielding

$$
\sigma(r, t)=w_{0}(r) \exp \left\{-g_{1} \int_{0}^{t} \chi(r, s) \mathrm{d} s\right\} .
$$

Here the initial condition is $\sigma(r, 0)=w_{0}(r)$.

Finally, we can express the solutions by matching across the region boundaries. We note that the upper curve of separation can be expressed as

$$
t=T(r)=R^{-1}(r)
$$

and the lower as

$$
t=T_{1}(r)=R^{-1}(r-h)
$$

Then we work upwards to establish

$$
\begin{aligned}
\sigma_{A}(r, t) & =w_{0}(r) \exp \left\{-g_{2} t\right\}, \\
\sigma_{B}(r, t) & =f_{1} \int_{0}^{t}\left[\chi(r, s) \exp \left\{-\left(f_{1}+g_{1}\right) \int_{s}^{t} \chi(r, \tau) \mathrm{d} \tau\right\}\right] \mathrm{d} s \\
& +w_{0}(r) \exp \left\{-\left(f_{1}+g_{1}\right) \int_{0}^{t} \chi(r, s) \mathrm{d} s\right\}, \\
\sigma_{C}(r, t) & =w_{0}(r) \exp \left\{-g_{1} \int_{0}^{t} \chi(r, s) \mathrm{d} s\right\}, \\
\sigma_{D}(r, t) & =\sigma_{B}(r, T(r)) \exp \left\{-g_{2}(t-T(r))\right\}, \\
\sigma_{E}(r, t) & =f_{1} \int_{T_{1}(r)}^{t}\left[\chi(r, s) \exp \left\{-\left(f_{1}+g_{1}\right) \int_{s}^{t} \chi(r, \tau) \mathrm{d} \tau\right\}\right] \mathrm{d} s \\
& +\sigma_{C}\left(r, T_{1}(r)\right) \exp \left\{-\left(f_{1}+g_{1}\right) \int_{T_{1}(r)}^{t} \chi(r, \tau) \mathrm{d} \tau\right\},
\end{aligned}
$$


and

$$
\sigma_{F}(r, t)=\sigma_{E}(r, T(r)) \exp \left\{-g_{2}(t-T(r))\right\}
$$

We eschew the straightforward expression for the corresponding tensions, as being non-informative.

\section{Serial Elasticity}

The above model reflects the historical form up until 1969, in that it allowed for elasticity in the system only in the cross-bridge tails. From the beginning it was recognized that the upwardly scaled full muscle model should be corrected for the elasticity of the attaching tendons. The elasticity of the thick and thin filaments and the other attaching structure in the sarcomere likewise was nominally acknowledged but usually it was supposed, if only for convenience in modeling, that the contribution of the stretch of the filaments was negligible compared to that of the cross-bridges. Only in 1994, after experiments ([17 and [19]) confirmed that only 30 to $50 \%$ of the compliance of the filament should be ascribed to the cross-bridge tails did this attitude begin to change.

Introducing this contribution in the Huxley model is important. A) If the attachment rate of the cross-bridges depends upon the number of myosin heads at a given extension which find themselves adjacent to a binding site on the actin fiber, the probabilities of attachment must change with tension, since the elastic stretch of the thin and thick filaments must differ. B) There are purely mechanical effects. First, the long-standing way of determining the elastic modulus of the cross-bridge tails is through experiments measuring the force response as super-posed small extensions are applied to tetanically-contracting muscles. If part of the measured deformation is due to elasticity of the filaments, then the previous estimates of elasticity of the cross-bridge tails would have been lower than actually was true, and in turn this would cause inaccuracies in predictions of response in other experiments. Similarly, once the cross-bridge-tail elastic modulus has been deduced, the most convenient way to determine evolution of the numbers of cross-bridges attached at a given time in an experiment is to measure the force response under such super-posed extensions of the muscle. If the elasticity were all in the cross-bridges, the force would be proportional to the number attached, if not, as we see below, this proportionality fails. Finally, the filament elasticity must contribute to the form of the force-time curve in a time-varying experiment, as during an excitation or relaxation or during a forced movement. 
Direct inclusion of the kinetic effect A) is naturally done through change of the attachment and detachment rules $F$ and $G$, or in more elaborate multi-state kinetics through change in the kinetic coefficients. Most of the literature on compliance effects focuses on this aspect. Here we will consider the mechanical effect B), retaining the original Huxley kinetics. The bibliography includes articles treating both effects, but here we discuss only those which include significant discussion of the mechanical effects.

In 1969 Julian [5] did calculations with a truncated Huxley model, including a non-linear elastic element in series with the contractile element. He also introduced a time-course excitation for tetanus and twitch. Following the 1994 experiments, Goldman and Huxley [16] did rough computations overlying the model, extended somewhat in 1996 by Huxley and Tideswell [21]. Using a discretized version of the model in 1993 and 1994 Luo, Cooke and Pate [?, [18] considered the effect of series compliance on decay of tetanus and on the lead-lag problem discussed below. In 1996 Mijailovich, Fredberg and Butler [22] considered a detailed model with elasticity in both actin and myosin, decoupling the equations of deformation from the cross-bridge kinetics to obtain computations of the lead-lag problem similar to those below. Torelli in 1997 [24] established existence and uniqueness for the fully coupled Huxley-kinetics, elastic actin problem with rather general constitutive equations.

Here we integrate the standard Huxley model with an added series linear elasticity to illustrate the effect of non-cross-bridge elasticity 7 . We thus can use the integrated density equations $[26$, but need to reexamine the evolution of the displacement.

Let $L+l(t)$ denote the total length of the muscle/series-elastic element, with $l(0)=0$. If the length of the series-elasticity element is $l_{s}(t)$ and that of the contractile unit $l_{m}(t)$, we have

$$
l_{s}^{\prime}(t)+l_{m}^{\prime}(t)=l^{\prime}(t)
$$

The contraction velocity of the muscle then is

$$
v(t)=-l_{m}^{\prime}(t)=l_{s}^{\prime}(t)-l^{\prime}(t)
$$

The length of the elastic unit, or spring, is determined by the tension generated by the muscle, and hence carried by the elastic unit, as

$$
l_{s}(t)-L_{s}=P(t) / E_{s} ;
$$

\footnotetext{
${ }^{7}$ The elaboration which includes detailed actin and myosin elasticity does not lead to improvements in the output.
} 
where $L_{s}$ is the rest-length of the spring and $E_{s}$ its modulus of elasticity ${ }^{8}$ From 28) we have a relation between the cross-bridge extension and the force in the spring:

$$
\begin{aligned}
\chi(r, t) & =r-\int_{0}^{t} v(s) \mathrm{d} s=r-\left(l_{s}(t)-l_{s}(0)\right)+l(t) \\
& =r-\frac{1}{E_{S}}(P(t)-P(0))+l(t)
\end{aligned}
$$

Now we combine (30) and [16, the representation for $P(t)$, to find

$$
\chi(r, t)=r-\left(\frac{U E_{m}}{E_{s}}\right)\left[\int_{-\infty}^{\infty} \chi(p, t) \sigma(p, t) \mathrm{d} p-\int_{-\infty}^{\infty} p w_{0}(p) \mathrm{d} p\right]+l(t) .
$$

Here we recall the notation $w_{0}$ for the initial distribution of attached cross-bridges.

To simplify calculations, let us set

$$
\begin{aligned}
\mu & =\frac{U E_{m}}{E_{s}}, \\
Q(t) & =\int_{-\infty}^{\infty} \sigma(p, t) \mathrm{d} p, \\
M(t) & =\int_{-\infty}^{\infty} \chi(p, t) \sigma(p, t) \mathrm{d} p .
\end{aligned}
$$

In light of (17), $\mu$ is the ratio of a "maximal" elastic modulus of the contractile unit to the elastic modulus of the serial unit; $U Q(t)$ is the number of attached cross-bridges.

Using these abbreviations, 31 is

$$
\chi(r, t)=r-\mu(M(t)-M(0))+l(t)
$$

We multiply through by $\sigma(r, t)$ and integrate to obtain

$$
M(t)=\int_{-\infty}^{\infty} r \sigma(r, t) \mathrm{d} r-\mu[M(t)-M(0)] Q(t)+l(t) Q(t),
$$

and solve to find

$$
M(t)-M(0)=\left[\int_{-\infty}^{\infty} r \sigma(r, t) \mathrm{d} r-M(0)+l(t) Q(t)\right] /(1+\mu Q(t)) .
$$

\footnotetext{
${ }^{8}$ To facilitate comparison to the previous results for the Huxley57 model, we assume linear elastic response for the series unit and for the elasticity of the cross-bridges. Changing to nonlinear elastic forms leads to obscuring complexity
} 
Finally, then, we obtain the prescription for the displacement

$$
\chi(r, t)=r-\frac{\mu}{1+\mu Q(t)} \int_{-\infty}^{\infty}\left(\sigma(p, t)-w_{0}(p)\right) p \mathrm{~d} p+\frac{l(t)}{1+\mu Q(t)} .
$$

We now can use (38) to calculate $\chi$ for the formal solutions (26), and also to determine the region boundaries in Fig 6 for the current case. In practice, it is necessary to calculate numerically. The results shown below are found by a front-tracking scheme: the fronts in the $r-t$ plane are those where the attachment and detachment functions change form: $\chi(r, t)=0$ and $\chi(r, t)=h$. Given these $r$ values at a specified time, we project them forward for a time-step, using the current velocity. Then the resulting values of $\sigma$ at the new time are calculated from 26. These values are then used to find a new $\chi$, from 38 and the process continued.

\section{Isometric Loading; Tetanus and Twitch}

In this instance $l(t)=0$ and the initial density of attached cross-bridges is assumed to be zero. Computations were made for various values of the compliance ratio

$$
\mu=\frac{U E_{m}}{E_{s}} .
$$

The computation for $\mu=0$ corresponds to rigid attachments of the muscle unit and increasing values of $\mu$ describe increasing compliance in the serial element.

The first set of computations is to verify that the presence of serial compliance changes the timing of the number and tension development in a tetanus. In Figure 7 graphing normalized values of both quantities, we see that at a value of $\mu=0$ (original Huxley model), the tension does lead the number, while with serial compliance, here $\mu=10$, the precedence is reversed. It also is worth noting that the presence of compliance significantly delays the development of both number and tension. The point at which lead becomes lag is on the order of $\mu=2$.

These computations show that contrary to some conjectures in the literature, the shift in tension-number development can be the effect simply of serial compliance.

As pointed out above, however, the experimentalists compare instead the evolution of the tension and the stiffness (modulus of elasticity) of the muscle 9 .

\footnotetext{
${ }^{9}$ To examine the stiffness experimenters apply very high frequency superposed extensions ( $c f$., e.g., [26]).
} 

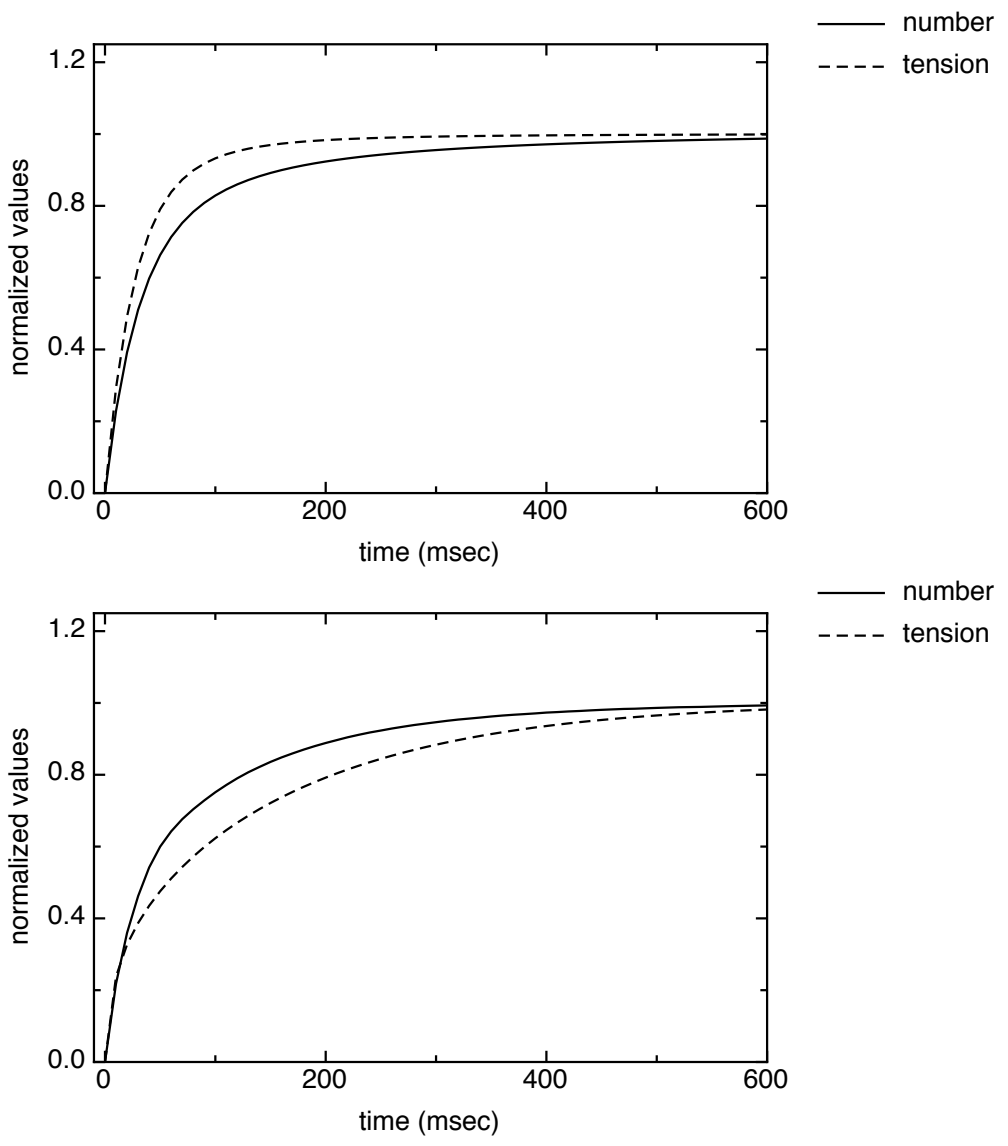

Figure 7: Predictions of development of (normalized) number and tension, without (upper graph) and with serial compliance $(\mu=10)$ 

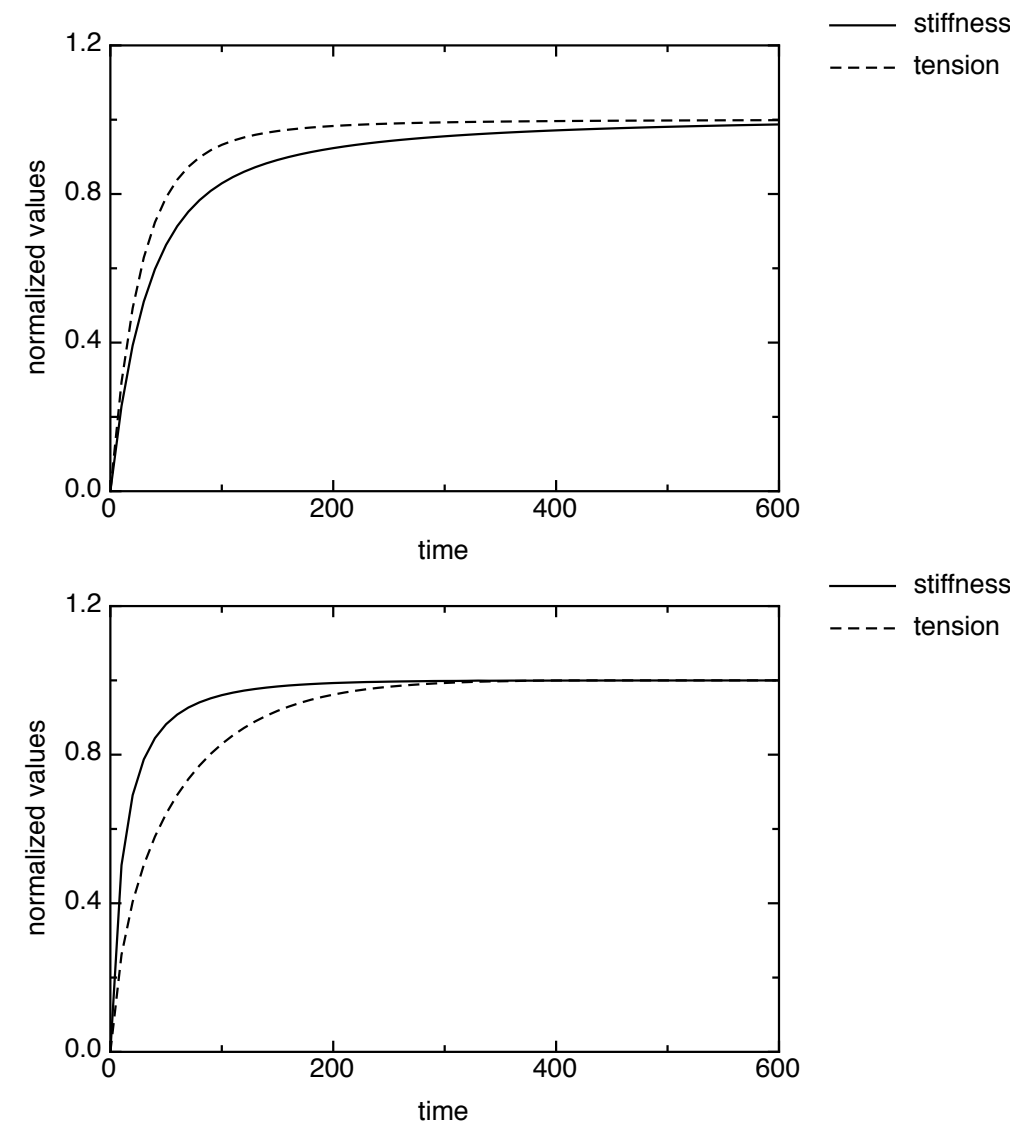

_ stiffness

- - - tension

Figure 8: Predictions of development of (normalized) stiffness and tension, without (upper graph) and with serial compliance $(\mu=3)$

To examine this we combine (16) and (37) to obtain

$$
\begin{aligned}
P(t)= & U E_{m}\left\{M(0)+\left[\int_{-\infty}^{\infty} r \sigma(r, t) \mathrm{d} r-M(0)\right] /(1+\mu Q(t))\right\} \\
& +U E_{m} \frac{Q(t)}{1+\mu Q(t)} l(t)
\end{aligned}
$$

Thus the modulus of elasticity in an increment of length is $U E_{m} \frac{Q(t)}{1+\mu Q(t)}$. The normalized values of this quantity are plotted together with the tension in Figure 8. Again, the change from leading to lagging occurs, notably at a much lower value of $\mu$ (here the breaking point is $\mu=0.5$ ).

Next we turn to the effect of compliance on a twitch. Hill in [2] produced a series of twitches on the same muscle, first with rigid attachments, then with 

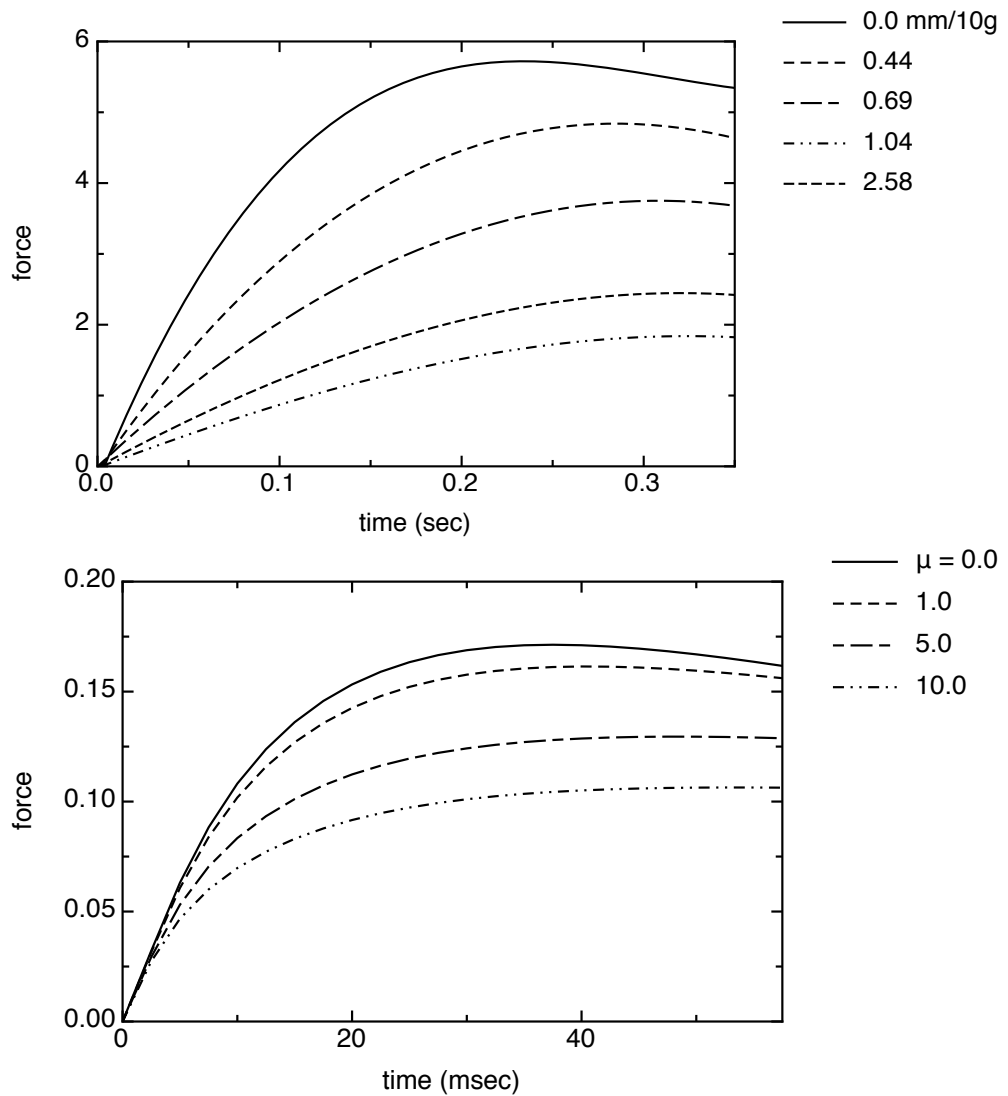

$-\mu=0.0$

$---1.0$

$---5.0$

$-\cdots+10.0$

Figure 9: Hill's compliant twitches (top) and calculated compliant twitches

increasingly soft springs for one of the end attachments. His data are replicated in Figure 9. Using an double-exponential excitation function proposed by Julian $[5]^{10}$ we use the above method to obtain the calculated results shown in the second graph in the figure. The details are not the same ${ }^{11}$, but the fundamental behavior, that the peak response is lowered in magnitude and shifted to the right, is observed.

\footnotetext{
${ }^{10}$ The coefficient $f_{1}$ is modified by multiplying by this support function.

${ }^{11}$ In particular, the time scale is greatly different. The rate coefficients for muscle increase exponentially with temperature, and limits of the instrumentation of the time required Hill to perform his tests at freezing temperatures in order to be able to capture the experimental traces. See [3, 13
} 


\section{References}

[1] A. V. Hill. The heat of shortening and the dynamic constants of muscle. Proc. R. Soc. Lond. B, 126:136-195, 1938.

[2] A. V. Hill. The effect of series compliance on the tension developed in a muscle twitch. Proc. R. Soc. Lond. B, 138:325-329, 1951.

[3] A. V. Hill. The influence of temperature on the tension developed in an isometric twitch. Proc. R. Soc. Lond. B, 138:349-354, 1951.

[4] A. F. Huxley. Muscle structure and theories of contraction. Prog. Biophys. Biophys. Chem., 7:255-318, 1957.

[5] F. Julian. Activation in a skeletal muscle contraction model with a modification for insect fibrillar muscle. Biophys. J., 9:547-570, 1969.

[6] J. Thorson and D. C. S. White. Distributed representations of actin-myosin interaction in the oscillatory contraction of muscle. Biophys. J., 9:360-389, 1969.

[7] A. F. Huxley and R. M. Simmons. A quick phase in the series-elastic component of striated muscle, demonstrated in isolated fibres from the frog. $J$. Physiol., 208:52P-53P, 1970.

[8] G. I. Zahalak. A distribution-moment approximation for kinetic theories of muscular contraction. Math. Biosci., 55:89-114, 1981.

[9] G Cecchi, P. J. Griffiths, and S. Taylor. Muscular contraction: Kinetics of crossbridge attachment studied by high-frequency stiffness measurements. Science, 217:70-72, 1982.

[10] H. Sugi and T. Kobayashi. Sarcomere length and tension changes in tetanized frog muscle fibers after quick stretches and releases. Proc. Natl. Acad. Sci., 80:6422-6425, 1983.

[11] G. Avanzolini and A. Cappello. The characteristic method applied to the study of muscle dynamics. Bul. Math. Biol., 46:827-844, 1984.

[12] W. O. Williams. On the Lacker-Peskin model for muscular contraction. Math. Biosci., 70:203-216, 1984.

[13] A. F. Bennett. Temperature and muscle. J. Exp. Biology, 115:333-344, 1985. 
[14] M. A. Bagni, G. Cecchi, and M. Schoenberg. A model of force production that explains the lag between crossbridge attachment and force after electrical stimulation of striated muscle fibers. Biophys. J., 54:1105-1114, 1988.

[15] F. E. Zajac. Muscle and tendon: properties, models, scaling, and applications to biometrics and motor control. Crit. Rev. in Biomed. Eng., 17:359411, 1989.

[16] Y. E. Goldman and A. F. Huxley. Actin compliance: are you pulling my chain? Biophys. J., 67:2131-2133, 1994.

[17] H. E. Huxley, A. Stewart, H. Sosa, and T. Irving. X-ray diffraction measurements of the extensibility of actin and myosin filaments in contracting muscle. Biophys. J., 67:2411-2421, 1994.

[18] Y. Luo, R. Cooke, and E. Pate. Effect of series elasticity on delay in development of tension relative to stiffness during muscle activation. Am. J. Physiol., 267:C1598-C1606, 1994.

[19] K. Wakabayashi, Y. Sugimoto, H. Tanaka, Y. Ueno, Y. Takezawa, and Y. Amemiya. X-ray diffraction evidence for the extensibility of actin and myosin filaments during muscle contraction. Biophys. J., 67:2422-2435, 1994.

[20] D. A. Smith and M.A. Geeves. Strain-dependent cross-bridge cycle for muscle. Biophys. J., 69:524-537, 1995.

[21] A. F. Huxley and S. Tideswell. Filament compliance and tension transients in muscle. J. Muscle Res. Cell Motil., 17:507-511, 1996.

[22] S.M. Mijailovich, J.J. Fredberg, and J.P. Butler. On the theory of muscle contraction: filament extensibility and the development of isometric force and stiffness. Biophys. J., 71:1475-1484, 1996.

[23] M. Forcinito, M. Epstein, and W. Herzog. Theoretical considerations on myofibril stiffness. Biophys. J., 72:1278-1286, 1997.

[24] A. Torelli. Study of a mathematical model for muscle contraction with deformable elements. Rend. Sem. Mat. Univ. Politec. Torino, 55:241-271, 1997.

[25] T. L. Daniel, A. C. Trimble, and P. B. Chase. Compliant realignment of binding sites in muscle: Transient behavior and mechanical tuning. Biophys. J., 74:1611-1621, 1998. 
[26] M. A. Bagni, G. Cecchi, B. Colombin, and F. Colomo. Sarcomere tensionstiffness relation during the tetanus rise in single frog muscle fibres. J. Muscle Res. Cell Motil., 20:469-476, 1999.

[27] T. A. J. Duke. Molecular model of muscle contraction. Proc. Natl. Acad. Sci., 96:2770-2775, 1999.

[28] U. Proske and D. L. Morgan. Do cross-bridges contribute to the tension during stretch of passive muscle? J. Muscle Res. Cell Motil., 20:433-442, 1999.

[29] G. Wang, W. Ding, and M Kawai. Does thin filament compliance diminish the cross-bridge kinetics? a study in rabbit psoas fibers. Biophys. J., 76:978984, 1999.

[30] M. A. Bagni, G. Cecchi, B. Colombini, and F. Colomo. A non-cross-bridge stiffness in activated frog muscle fibers. Biophys. J., 82:3118-3127, 2002.

[31] D. Martyn, P. Chase, M. Regnier, and A. Gordon. A simple model with myofilament compliance predicts activation-dependent crossbridge kinetics in skinned skeletal fibers. Biophys. J., 83:3425-3434, 2002.

[32] E. J. Perreault, S. J. Day, Hulliger, C. J. Heckman, and T. G. Sandercock. Summation of forces from multiple motor units in the cat soleus muscle. J. Neurophysiol., 89:738-744, 2003.

[33] P. B. Chase, J. M. Macpherson, and T. L. Daniel. A spatially explicit nanomechanical model of the half-sarcomere: Myofilament compliance affects ca2+-activation. Ann Biomed. Eng, 32:1559-1568, 2004.

[34] N. P. Smith, C. J. Barclay, and D. S. Loiselle. The efficiency of muscle contraction. Prog. Biophys. Mol. Bio., 88:1-58, 2005.

[35] KS Campbell. Filament compliance effects can explain tension overshoots during force development. Biophys. J., 91:4102-4109, 2006.

[36] M. Kawai and H. R. Halvorson. Force transients and minimum cross-bridge models in muscular contraction. J. Muscle Res. Cell Motil., 28:371-395, 2007.

[37] Y. C. Fung. Biomechanics : mechanical properties of living tissues. SpringerVerlag, New York, 1993.

[38] J. Keener and N. Sneyd. Mathematical Physiology, chapter 18, pages 542578. Springer, NY, 1998. 
[39] C. S. Peskin. Mathematical Aspects of Heart Physiology. Courant Institute Lecture Notes. Courant Institute, 1975. 